\title{
EFFICIENCY THAT BORDERS ON THE IMPOSSIBLE ELECTROHYDRAULIC DRIVES
}

\author{
Dipl.-Ing.(FH) Walter List
}

Head of Product Development, WEBER-HYDRAULIK GMBH, Emil-Weber-Platz 1, A-4460 Losenstein, Österreich Tel.: +43 72556237 12125; E-mail address: walter.list@weber-hydraulik.com

\begin{abstract}
Hydraulic displacement systems are a technology that has been known for many years. Nevertheless, the impression is created that the large fields of application are not only being developed very tentatively, but in many cases are being completely denied. What might be the reason for this? Are the electromechanical solutions better? What is the missing element to build the bridge from the available hydraulic-mechatronic technology to broad customer application?

This paper provides an overview of how the targeted use of the latest technologies in the product and in the entire value-added chain has resulted in a complete mechatronic hydraulic system that pushes the limits of feasibility in terms of power, accuracy and efficiency and delivers a clearly calculable benefit for both the plant manufacturer and the end customer. In addition to the great accuracy and high reliability, there are also considerable savings in the total cost of ownership (TCO) due to a previously unattained level of efficiency.

The thoughts of health and environmental protection are just as much a part of the considerations as product safety and personal protection.

Finally, an outlook on possible expansions is given, and which areas of application are possible for the technologies developed within the project. The aim is not to show how the traditional fields of application of hydraulics are to be defended, but rather to use the strengths of a hydraulic-mechatronic approach in a conscious and clearly structured way to make new applications possible.
\end{abstract}

Keywords:linearactuator, servohydraulic, elektrohydraulic drive

\section{INTRODUCTION}

"Over 80 years of experience in hydraulics make WEBER-HYDRAULIK a competent specialist for hydraulic drive and control technology. This is our obligation".

The WEBER-HYDRAULIK Group now comprises seven locations. Together they stand for tailor-made and functionally reliable hydraulic solutions. The aim of bundling expertise is to be able to offer successful solutions in the demanding and challenging market of mobile and stationary hydraulics.

"Our investments in research, development and modern production technologies as well as the continuous qualification of our employees ensure that we have the short path to progress".

The development of high-quality cylinders, control blocks, valves and power units has a long tradition in the group of companies and is therefore our technological core competence.
Building on this, the Group also offers its customers the realisation of sophisticated application-specific system solutions as a leading international, innovative and future-oriented partner. 


\section{EHA-ELEKTROHYDRAULIC ACTUATOR}

\subsection{Initial situation}

Hydraulic linear units are a technology known for many years and have significant advantages compared to electromotive drives and electric linear drives. Where large forces, precise movements and high efficiency are required, the electro-hydraulic linear actuator represents a technology with almost no alternatives. Nevertheless, sometimes the impression is given that the very large applications are only very tentatively being developed.

WEBER-HYDRAULIK has been developing customer-specific electro-hydraulic and servohydraulic linear axes for several years and supplies them to well-known companies in the automotive, mechanical engineering and plant construction industries.

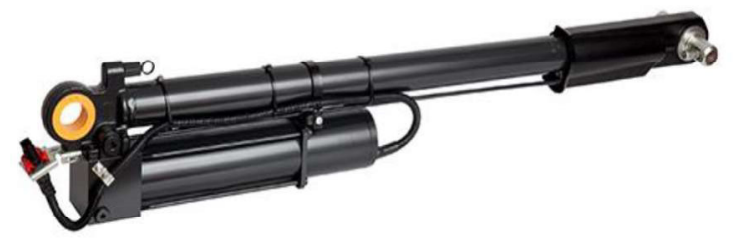

Figure 2: compact linear actuator for mobile use (WEBER HYDRAULIK)

The specifications range from electro-hydraulic compact units for the automotive industry, through resolved systems for industrial applications, to compact linear drives in highly dynamic machine axes. The fields of application are just as broad as the customer segments.

The present work will be limited to industrial applications. These axes are manufactured in the Austrian plant of WEBER-HYDRAULIK, in Losenstein, Upper Austria. The electro-hydraulic axes for mobile applications are developed and manufactured at the sites in Güglingen (Heilbronn, Germany) and Veenendaal (Utrecht, Netherlands).

\subsection{Theoretical aspects, classification}

Electrohydraulics vs. electromechanics - a comparison of unequal technologies.

Electromechanics and electrohydraulics are not mutually exclusive systems.

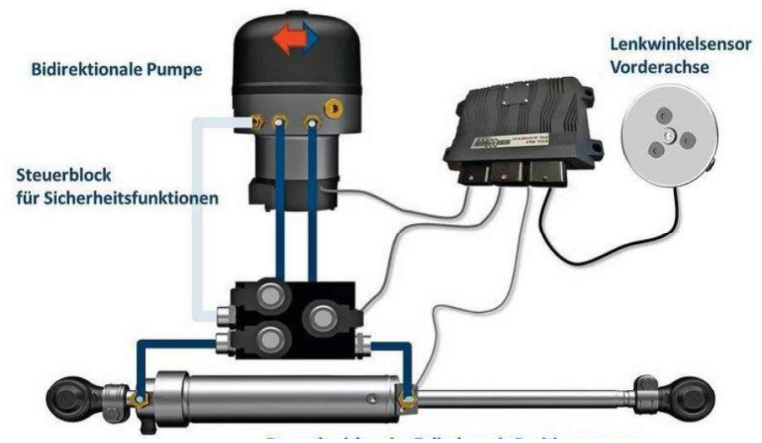

Figure 1: linear actuator (WEBER HYDRAULIK)

Many applications use these technologies in a complementary manner, whereby electrohydraulic drives fully exploit their advantages when large forces are applied. The main decision criterion for the use of an electrohydraulic actuator is therefore usually the high forces required with good efficiency. Pneumatic cylinders and electromechanical axes are advantageous for very small loads during operation. In terms of energy technology, however, pneumatics also have major deficits

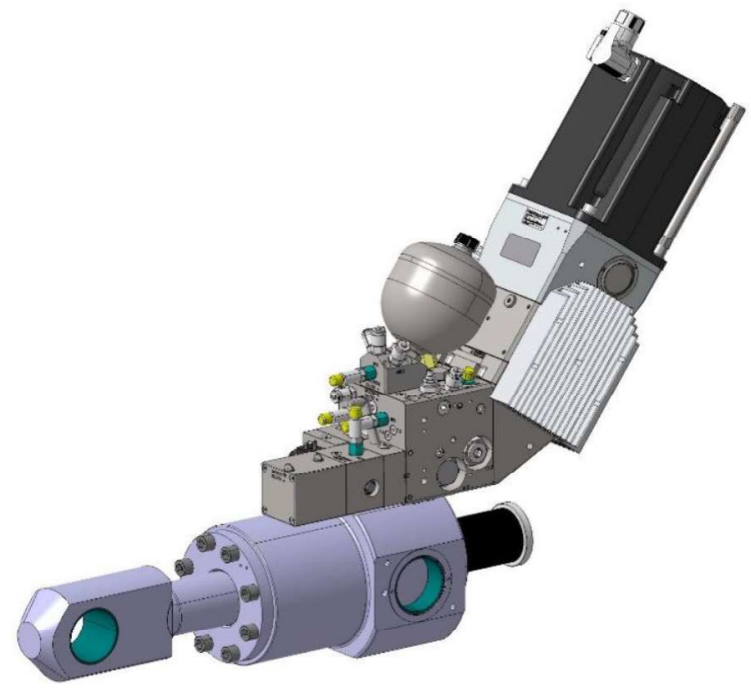

Figure 3: compact linear actuator for industrial purposes (WEBER HYDRAULIK)

here. Only about $1 / 3$ of the primary energy used is converted into power by pneumatics. For electromechanical drives, a primary energy utilization of about $80 \%$ can be assumed. Depending on the drive solution (ball screw, 
roller screw, ...) and the forces generated, the values vary greatly and can also be significantly lower.

The figure shows a summary of the system boundaries of the individual alternatives. Electric axes are a viable solution with good efficiency components makes the whole much more than the sum of the individual components.

In order to produce an application-specific optimized EHA, it is important that all components are optimally matched to each other. Even if the performance data of a pump, a

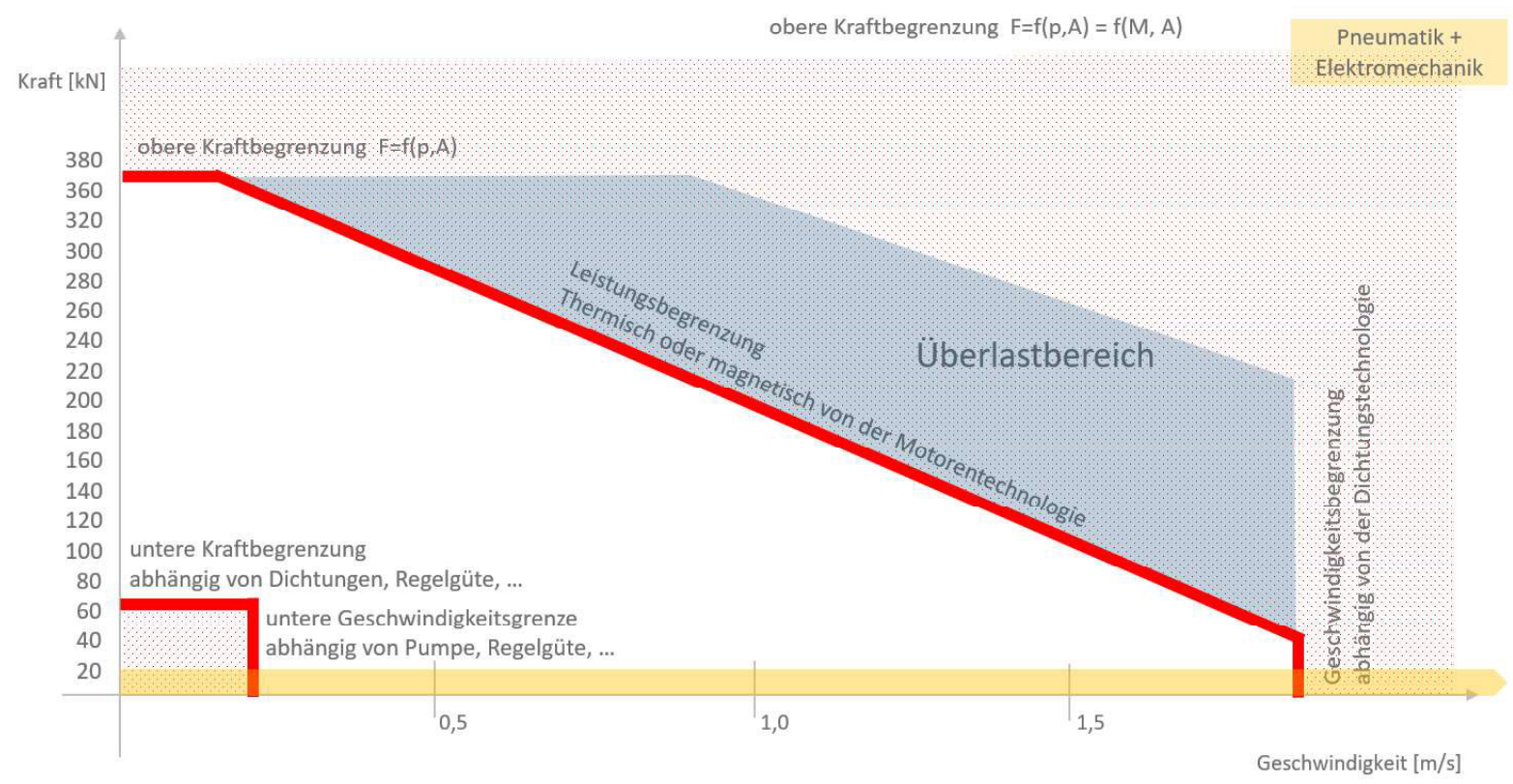

Figure 4: application area hydraulic linear actuator

values at low forces. Likewise, very high speeds and strokes can be achieved with electric axes at very low forces (e.g. toothed belt axes). At higher forces, electromechanical axes quickly reach system-related limits, and the speeds and dynamics are then relatively low due to the performance.

When high forces, high dynamics, high precision and high efficiency are combined in the specifications, the advantages of the electrohydraulic axis come into their own. The relatively low rotational masses allow very high dynamics. The robustness of the hydraulics allows high overloads in the short term. When using high-quality measuring systems, the EHA also achieves an exceptionally high, hardly expected precision that is not inferior to electromechanical axes.

\section{EFFICIENCY CONSIDERATIONS EHA'S}

Hydraulic linear actuators are not classic hydraulic components such as pumps, cylinders and valves, which are procured from a catalogue program. Rather, they are mechatronic systems in which the precise coordination of the individual cylinder and a drive motor can be derived with simple mathematics, the real overall performance of the EHA is difficult to determine and calculate analytically. This calculation becomes almost impossible when working with highly fluctuating, different load and operating profiles. Currently, only insufficient numerical methods are available which are suitable, for example, for predicting the overall efficiency of the EHA's in a specific application. Here WEBER HYDRAULIK takes a pragmatic approach and typically tests EHA's together with the end customer in the final application by means of a practical test. Strictly speaking, there is no better way to prove functionality and efficiency than in the customer application. This involves measuring the total energy consumption in the application under many other parameters.

The main design issues and problems that arise in this context will be briefly discussed below.

\subsection{Motors}

Modern motors, especially servo motors, have quite high efficiencies. Due to the possibility of short-term multiple overloads, it is possible to 
operate the motors in a range that is significantly higher than the rated power, but at which quite useful operating values are obtained for the EHA designer of valves is also in a commercial and technical field of tension. Large valves have large actuating forces and are expensive. Small valves

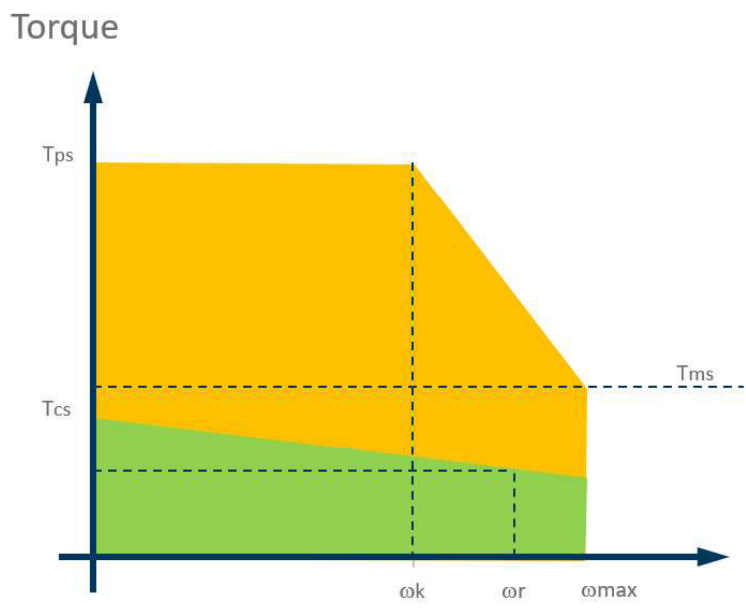

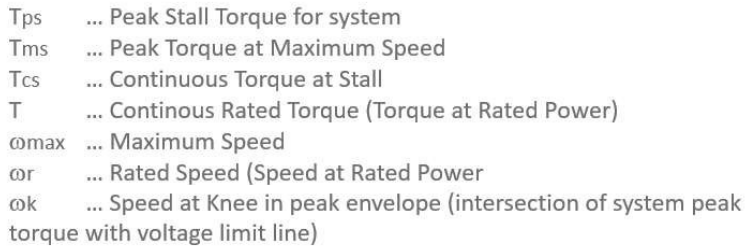

Continuous Duty

Intermittent Duty

Figure 5: application map servo motor

system.

For the design of the motors themselves it is often not the last percentage point of nominal efficiency that is important. For particularly dynamic applications, it is often a question of the mass moment of inertia and the acceleration capability of the system. In spite of all these theoretical considerations, it is hardly possible to calculate the motor efficiency reasonably using a dynamic application. The same considerations regarding the load profile apply here as for the overall axis.

Overall, the designer's influence on the exact motor characteristics is very small in reality, since modern motors are usually purchased as catalogue goods. For simple applications where dynamics and efficiency are not the main focus, we also select more cost-effective motor concepts, such as speed-controlled DC motors with a control system adapted to the vehicle electrical system. The variety of variants here is almost unmanageable.

\section{2. valves}

The influence of valves on the overall efficiency of hydraulic equipment is undisputed. A reduction in flow losses inevitably leads to a higher efficiency of the overall system. In principle, this thesis is clearly understandable and does not need further explanation. However, the have small actuating forces and are cheaper.

For reasons of system safety, load holding valves are also necessary in many cases. This is mainly used to enable the machine manufacturer to maintain the appropriate safety level (SIL) in order to achieve $\mathrm{CE}$ conformity with the machines and devices. In connection with functional safety, there are many issues that are not discussed here in detail.

If these valves, which are usually generously dimensioned in the flow, also have to switch quickly - the switching time is usually less than 50 milliseconds and directly affects the dynamics of the application - large, fast switching solenoids are required. Since there is typically little oil in the closed system EHA, the pilot control of valves can become a challenging design task. This is where it is of great advantage within the WEBER-HYDRAULIK Group to have access to

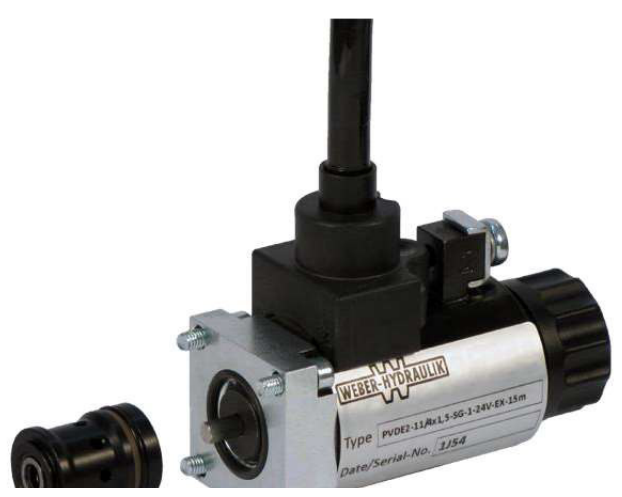

Figure 6: cartridge valve (WEBER HYDRAULIK) 
the appropriate valve know-how in order to solve such tasks accurately.

\section{3. cylinders}

Cylinders have been part of our core business for many decades. At the Austrian location of the WEBER-HYDRAULIK Group alone, more than 160,000 cylinders are manufactured annually.

Hydraulic cylinders are technologically already highly exhausted and their efficiency is difficult to improve. The tribological systems sealcylinder wall and seal-rod surface are the main factors influencing the overall efficiency of a hydraulic cylinder. There is a large number of scientific papers on this subject (it is beyond the scope of this paper to present the overall context here). When using commercially available sealing systems, there is little room for maneuver at this point anyway due to the specifications of the seal manufacturers.

Our decades of experience have shown that the efficiency of hydraulic cylinders with a piston diameter of approx. $100 \mathrm{~mm}$ reaches a value of more than $98 \%$.

An exception to this is servo cylinders with hydrostatic rod guidance and without contacting seals. However, these are used almost exclusively in extremely dynamic special applications, such as vibration testing. Here, even greater mechanical efficiencies are achieved, but there are also high leakage oil losses through the gap seals.

\section{4. pumps}

The best energy saved is the energy that was not generated.

The pump, as a hydraulic energy generator, is therefore one of the most critical components in terms of the overall efficiency of the EHA.

The pump efficiency is composed as follows:

$$
\begin{aligned}
& \eta_{\text {ges }}=\eta_{v o l} \cdot \eta_{h m} \\
& \eta_{v o l}=\frac{Q}{n \cdot V_{t h}} \\
& \eta_{h m}=\frac{\eta_{g e s}}{\eta_{v o l}}=\frac{\Delta p \cdot Q}{M \cdot 2 \cdot \pi \cdot n} \cdot \frac{n \cdot V_{t h}}{Q}=\frac{\Delta p \cdot V_{t h}}{2 \cdot \pi \cdot M} \\
& P_{\text {mech }}=M \cdot 2 \cdot \pi \cdot n \\
& P_{h y d r}=\Delta p \cdot Q
\end{aligned}
$$

By simply looking at the formulas, it can be seen that the pump efficiency is dependent on the pressure difference, the torque and the speed. In the literature, therefore, the representation of pump efficiency as a function of pressure difference and torque is also a common way of representation.

The picture is taken from a test series in our company and shows the typical course of the efficiency of a high-quality hydraulic pump at a certain speed. It is also easy to see that an optimum is achieved at a certain pressure. In every servo-motor hydraulic pump drive, this simple representation is overlaid by the speed. In order to be able to fully evaluate a pump, the efficiency curve must be determined for each relevant speed range.

The pump manufacturers themselves are very reluctant to provide such precise information on

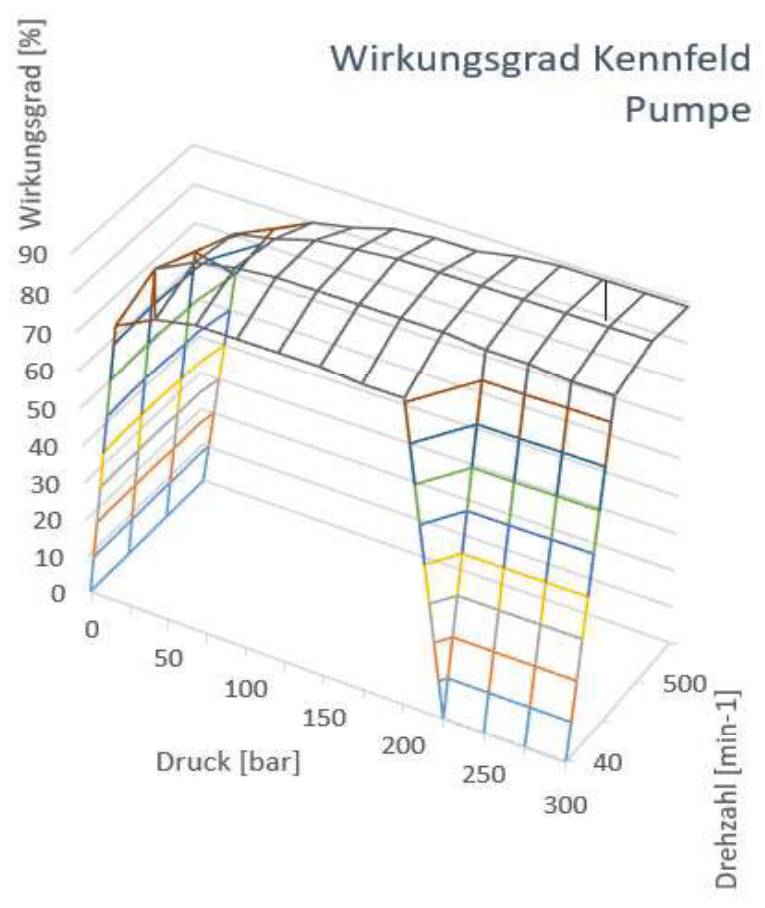

Figure 7: pump efficiency (WEBER-HYDRAULIK)

their products.

We have therefore carried out these tests on pumps from different manufacturers together with a partner close to the university and have drawn up corresponding characteristic diagrams. From the complexity of the interrelationships it can be concluded that a precise prediction of the 
overall efficiency, as already described, is almost

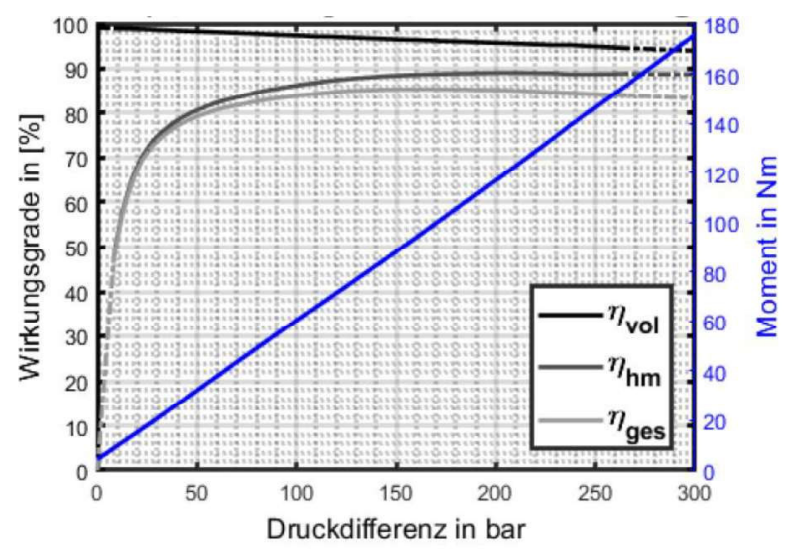

Figure 7 efficiency curve

impossible.

\section{5. elctronics, sensors and software}

A very important topic in the interaction of mechatronic systems are sensors and data acquisition devices. In most cases 2 different goals are pursued. On the one hand, it requires sensors that are fast and adapted to the application in terms of their accuracy to record the operating parameters required for the control. On the other hand, more and more data is collected which is not directly used for control but seems to be important for topics like predictive maintenance, industry 4.0 and IoT. There is currently a lot of hype here, with data often being collected without a precisely defined purpose.

Experience has shown that electro-hydraulic axes have a very specific technology base, strongly oriented towards existing customer experience. They are usually installed in an already existing environment and very often replace conservative hydraulic cylinders in existing machine concepts as part of an evolutionary development. This is due to the fact that in the vast majority of projects an already existing situation is improved. Thus, the largest projects in mechanical engineering are found in the replacement of servo valve solutions. Current experience shows us that linear actuators are less susceptible to failures than the servo valves that were replaced by the actuator. This is most likely due to the high long-term requirements on the purity of the operating medium. Here EHA's have a clear advantage due to the much more robust and less dirt sensitive design approach.

Due to the very specific development situation, it is understandable that users want to install their own, already known components in the devices. For the supplier of electro-hydraulic servo axes, this means that for the majority of applications there is no unconditional freedom in the selection of peripheral components. For sensors, filters, ... and many other small parts, it is necessary to implement the customer's specifications.

WEBER-HYDRAULIK also uses displacement sensors of various designs. Particularly good experience has been made with measuring systems that act directly on the piston rod. The advantage of a displacement measuring system that measures the actual position directly at the piston rod is primarily the rigidity and tolerance insensitivity of the measuring point. By eliminating manufacturing tolerances and effects based on the temperature response, very precise positioning and repeatability can be achieved.

In the high-precision drives, for example, we use linear measurement technology that directly uses electromagnetic field effects on the piston rod as a measurement signal. The measurement is made directly on the piston rod. This systematically excludes the entire tolerance chain of the EHA from the position measurement. The process for manufacturing these piston rods is complex and technologically very demanding. However, the effort is more than compensated by the positioning accuracy, which is extraordinarily precise for EHA.

\section{SUMMARY, USE, PROSPECT}

The technology "servo-hydraulic linear axis" has reached a very mature and industrial standard. Many systems are now in the field in various applications.

WEBER-HYDRAULIK provides its decades of experience in the design and production of high quality hydraulic components and develops sample systems in close cooperation with the customer. These sample systems are already tested in the customer's facilities and are then transferred into highly competitive series solutions within the framework of professional serialization. It goes without saying that these projects are carried out in accordance with recognised standards and development systems customary in the industry.

This ensures right from the start that the finished devices fit perfectly into the 
environment defined by the customer and that all project goals are achieved.

Today, revolutionary improvements are no longer to be expected for individual components. However, a big secret still lies in the precisely coordinated technology mix. Here, of course, the use of electronics in any form is a major future topic. However, the precise mechanical and hydraulic design and tuning of the components is the key competence that continues to lead to significant improvements. Predefined kits with predefined components are inevitably at a disadvantage. The apparent cost advantage of purchasing the application by using a modular system is lost, at least for highly dynamic applications in operation, due to the loss of the last percentage points of efficiency, dynamics and precision. Therefore, we have developed a technology construction kit for us, in which all necessary technologies are available. These are selected, combined, optimized and transformed into an inseparable hydraulic system together with the customer.

In the field of digital hydraulics there have been some promising approaches for quite some time. Especially in positioning, in load holding functions and in finest control tasks, the combination of servo-hydraulic drive and digitalhydraulic control technology seems to us to be an interesting combination which we will continue to pursue. Here, the near future will show whether new ideas and solutions from this field will be applied more widely in industry.

The integration of the individual disciplines will certainly lead to further improvements in the future. The extent to which pump, engine, valve technology and cylinder are merged into a single unit also depends on our customers and the respective applications and is difficult to estimate today.

It is to be expected that the actuators will also find their way into areas of application in which nature conservation and environmental protection play a major role. Due to the small quantities of oil in circulation, EHAs in compact design represent a very low risk to nature and the environment. An application is therefore also conceivable in environments where hydraulics are today viewed very critically.

By using our actuators, we have been enabling our customers for years to reduce the primary energy consumption of their applications by up to $75 \%$, depending on the load profile. Tons of CO2 have already been saved through the real application of the technology. Thus WEBERHYDRAULIK linear actuators are already making an active contribution to climate

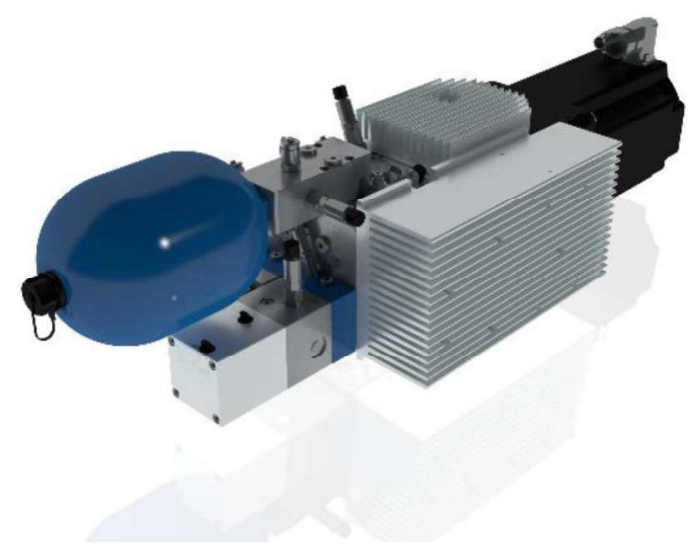

Figure 8 EHA (without cylinder)

protection and the reduction of greenhouse gases at our customers.

\section{COPYRIGHT}

This lecture text, the included pictures and tables are released by WEBER-HYDRAULIK GMBH and may be copied and passed on provided the source (WEBER-HYDRAULIK GMBH 2020) is mentioned. 